(1)

CrossMark

\title{
Pulmonary rehabilitation for bronchiectasis: if not now, when?
}

\author{
Mark L. Metersky ${ }^{1}$ and Richard L. ZuWallack ${ }^{2}$ \\ Affiliations: 'Division of Pulmonary, Critical Care and Sleep Medicine, UCONN Health, Farmington, CT, USA. \\ ${ }^{2}$ St Francis Hospital, Pulmonary and Critical Care, Hartford, CT, USA.
}

Correspondence: Mark L. Metersky, Division of Pulmonary, Critical Care and Sleep Medicine, UCONN Health, 263 Farmington Avenue, Farmington, CT 06030-1321, USA. E-mail: meterskyluchc.edu

@ERSpublications

This study provides robust evidence that outpatient pulmonary rehabilitation is effective in patients with bronchiectasis: it is time to increase the use of this under-utilised intervention

http://ow.ly/3tle30ndsZq

Cite this article as: Metersky ML, ZuWallack RL. Pulmonary rehabilitation for bronchiectasis: if not now, when? Eur Respir J 2019; 53: 1802474 [https://doi.org/10.1183/13993003.02474-2018].

Bronchiectasis, long considered an "orphan" disease, is now increasing in prevalence in both Europe [1] and North America [2]. Characterised by chronic cough with sputum production and permanently dilated airways [3], advanced disease may result in respiratory insufficiency due to airway obstruction. Many patients suffer from substantially impaired quality of life $[4,5]$, likely due to chronic respiratory symptoms (dyspnoea, cough, sputum production, wheeze), respiratory exacerbations, fatigue, exercise limitation, psychological symptoms, and constitutional manifestations of chronic infection and inflammation.

Remarkably, the only pharmacologic therapy supported by high-quality, randomised controlled trials in bronchiectasis is long-term, low-dose macrolide treatment; currently, there are no pharmacologic treatments approved for bronchiectasis by either the US Food and Drug Administration or the European Medical Agency. The 2017 European Respiratory Society guidelines for the diagnosis and treatment of bronchiectasis [6] assigned chronic macrolide therapy a conditional recommendation. Airway clearance, long considered an important component of bronchiectasis care, received a weak recommendation, based on low quality evidence. The only treatment that received a strong recommendation was for participation in a pulmonary rehabilitation programme.

Pulmonary rehabilitation, with patient-centred exercise training and collaborative self-management education delivered by an interdisciplinary team and centred on the specific needs of the patient [7], has evolved beyond the confines of chronic obstructive pulmonary disease (COPD) management. Pulmonary rehabilitation works through mitigating secondary impairments such as peripheral muscle dysfunction, psychosocial dysfunction, and maladaptive behaviours. These contribute to overall disease burden and are frequently treatable [8].

So, why a recommendation for pulmonary rehabilitation in bronchiectasis? The bronchiectasis guidelines included 14 trials in their pooled analyses [6], but many addressed airway clearance only. A recent systematic review including randomised or crossover trials of pulmonary rehabilitation or exercise training alone, found only four trials, with the review hampered by heterogeneity in design and totalling only 164 patients [9]. The analysis demonstrated significant, short-term improvements in exercise capacity and health-related quality of life, but these effects were not maintained at 6 months. Furthermore, there was no consistent improvement in cough-related quality of life in the pooled data from two studies [10, 11], with 
only one demonstrating improvement in the Leicester Cough Questionnaire score. Of potential importance, one trial of supervised exercise training plus a review of airway clearance therapy (versus standard therapy incorporating encouragement of regular exercise) demonstrated a reduced frequency of exacerbations and prolonged time to exacerbations in the treatment group [11]. Additionally, observational data from an inpatient programme supports pulmonary rehabilitation in bronchiectasis [12].

The study by PAtel et al. [13] in this issue of the European Respiratory Journal adds substantially to the evidence-base supporting pulmonary rehabilitation for bronchiectasis patients. In this observational study, bronchiectasis patients with a Medical Research Council (MRC) dyspnoea score of $\geqslant 2$ referred to pulmonary rehabilitation assessment clinics were prospectively recruited and given 8 weeks (16 supervised sessions) of outpatient pulmonary rehabilitation. Their clinical characteristics and rehabilitation outcomes were compared 1:1 to propensity-matched COPD patients who had previously completed pulmonary rehabilitation. Matching was based on age, gender, per cent of predicted forced expiratory volume in $1 \mathrm{~s}$ (FEV1), body mass index, MRC dyspnoea score and exercise capacity (assessed by the incremental shuttle walk (ISW) distance). Bronchiectasis patients with $\geqslant 10$ pack-year history of cigarette smoking or with a clinical diagnosis of COPD were excluded from the analysis. Both groups received exercise training in a programme fulfilling British Thoracic Society quality standards for pulmonary rehabilitation [14]. Study patients received educational sessions on numerous topics, including airway clearance. The outcomes measured after completion of the programme included completion rate, health-related quality of life assessed by the chronic respiratory disease questionnaire (CRQ), which includes domains of dyspnoea, fatigue, emotion and mastery, and the ISW distance, a measure of exercise capacity.

Over 5 years, 548 patients with a primary diagnosis of bronchiectasis were referred to pulmonary rehabilitation, with 213 of these included in the study; most exclusions were due to co-existing COPD. The groups were well-matched by the relevant variables. The bronchiectasis patients had a mean FEV1 of $68 \%$ of predicted and a median MRC dyspnoea score of 3. $74 \%$ completed the programme in each group; non-completion was mostly due to exacerbations. Post-intervention, both groups demonstrated significant improvements over baseline in the ISW distance and all four domains of the CRQ. Between-group changes in these variables, the primary outcomes of the study, were similar and not significantly different, with the exception of CRQ-fatigue, which improved more in the COPD group.

This study, therefore, supports the belief that patients with bronchiectasis stand to improve after completing pulmonary rehabilitation, with positive outcomes rivalling those found for COPD. Since the beneficial effects of pulmonary rehabilitation in COPD are well-established in clinical trials, including moderately large and clinically significant improvements in dyspnoea, exercise tolerance and quality of life [15], these are very important results. Perhaps dissemination of these positive findings among clinicians will increase referrals to pulmonary rehabilitation, as bronchiectasis was not listed among the five most common diseases leading to referral to European and North American centres, from a survey published in 2013 [16].

As is the case with most studies demonstrating important results, questions always arise. In this study, questions regarding generalisability come to mind. The median MRC dyspnoea score in the bronchiectasis patients was 3 on a 1-5 scale ("I walk slower than people of the same age on the level because of breathlessness or have to stop for breath when walking at my own pace on the level”) [17], higher than in two large studies of unselected bronchiectasis patients, in which it was 2 [18] and 1 [19]. Thus the study patients may not be representative of the broader population of bronchiectasis patients. For example, in a large research registry of bronchiectasis in the USA, 46\% did not report dyspnoea at rest or on exertion [20]. In these patients, other factors, such as cough, fatigue and exacerbations, may be more potent drivers of health-related quality of life. Exercise training provided to these patients may have fewer beneficial effects than in those with more dyspnoea burden.

While cough adds to the disease burden of bronchiectasis patients, the CRQ used in this study has no questions directly related to cough. However, the dyspnoea domain of the CRQ showed a greater effect size compared to the other three domains in a randomised, controlled trial of patients with mild to moderate bronchiectasis [21]. Furthermore, a meta-analysis of the psychometric properties of health-related quality of life instruments in bronchiectasis found dyspnoea to be an important predictor [22].

Another question raised by this study is: which intervention(s) resulted in the improvements observed? This is a perennial issue with pulmonary rehabilitation trials, since the components are typically offered as a package, not individually. All patients received both exercise training and education, including airway clearance training. While it likely that the exercise training was responsible for the improved exercise capacity, the basis of the improved quality of life is less intuitive, since effective airway clearance may improve both dyspnoea and quality of life in patients with bronchiectasis [23, 24]. Many patients with bronchiectasis are poorly adherent to airway clearance [20], so improved adherence may have contributed to the improvement in quality of life. 
As noted by the authors, a limitation of the current study is the lack of long term follow-up to determine whether the outcome improvements persisted. Based on experience with short-duration outpatient pulmonary rehabilitation of COPD patients, it would be anticipated that these benefits would wane over time [25], unless ongoing intervention was provided [26]. Potentially adding to this problem, many patients with bronchiectasis endure an extremely high treatment burden due to the use of time-consuming interventions, including airway clearance regimens and nebulised antibiotics. This introduces the possibility that they might be even less able than COPD patients to adhere to a regular exercise programme, simply due to time constraints. Furthermore, the social aspects of group exercise may be an important determinant of participation in regular exercise, yet some bronchiectasis patients are embarrassed by their cough and less likely to participate in group activities [27].

PAtel et al. [13] have provided robust evidence that bronchiectasis patients will adhere to an outpatient pulmonary rehabilitation intervention and, in the short term at least, will realise improvements in exercise capacity and health-related quality of life, similar to patients with COPD. Because of the research question they addressed, the researchers excluded patients with COPD. We know that COPD and bronchiectasis frequently overlap $[28,29]$. Indeed, 225 out of 548 patients $(41 \%)$ originally evaluated in the current study were excluded because of co-existing COPD or smoking history. Knowing that patients with either disorder stand to benefit from pulmonary rehabilitation, it is reasonable to assume that the large group with both processes should also improve. How the pulmonary rehabilitation intervention should be tailored to meet the needs of individuals with this overlap needs to be determined.

Conflict of interest: M.L. Metersky has nothing to disclose. R.L. ZuWallack reports advisory work for Philips Respironics, outside the submitted work.

\section{References}

1 Quint JK, Millett ER, Joshi M, et al. Changes in the incidence, prevalence and mortality of bronchiectasis in the UK from 2004 to 2013: a population-based cohort study. Eur Respir J 2016; 47: 186-193.

2 Seitz AE, Olivier KN, Adjemian J, et al. Trends in bronchiectasis among medicare beneficiaries in the United States, 2000 to 2007. Chest 2012; 142: 432-439.

3 McShane PJ, Tino G. Bronchiectasis. Chest 2018; in press [https://doi.org/10.1016/j.chest.2018.10.027].

4 Quittner AL, Marciel KK, Salathe MA, et al. A preliminary quality of life questionnaire-bronchiectasis: a patient-reported outcome measure for bronchiectasis. Chest 2014; 146: 437-448.

5 Wilson CB, Jones PW, O'Leary CJ, et al. Validation of the St. George's Respiratory Questionnaire in bronchiectasis. Am J Respir Crit Care Med 1997; 156: 536-541.

6 Polverino E, Goeminne PC, McDonnell MJ, et al. European Respiratory Society guidelines for the management of adult bronchiectasis. Eur Respir J 2017; 50: 1900629.

7 Spruit MA, Singh SJ, Garvey C, et al. An official American Thoracic Society/European Respiratory Society statement: key concepts and advances in pulmonary rehabilitation. Am J Respir Crit Care Med 2013; 188: e13-e64.

8 Nici L, ZuWallack R, Wouters E, et al. On pulmonary rehabilitation and the flight of the bumblebee: the ATS/ERS Statement on Pulmonary Rehabilitation. Eur Respir J 2006; 28: 461-462.

9 Lee AL, Hill CJ, McDonald CF, et al. Pulmonary rehabilitation in individuals with non-cystic fibrosis bronchiectasis: a systematic review. Arch Phys Med Rehabil 2017; 98: 774-82 e1.

10 Mandal P, Sidhu MK, Kope L, et al. A pilot study of pulmonary rehabilitation and chest physiotherapy versus chest physiotherapy alone in bronchiectasis. Respir Med 2012; 106: 1647-1654.

11 Lee AL, Hill CJ, Cecins $\mathrm{N}$, et al. The short and long term effects of exercise training in non-cystic fibrosis bronchiectasis--a randomised controlled trial. Respir Res 2014; 15: 44.

12 Zanini A, Aiello M, Adamo D, et al. Effects of pulmonary rehabilitation in patients with non-cystic fibrosis bronchiectasis: a retrospective analysis of clinical and functional predictors of efficacy. Respiration 2015; 89: 525-533.

13 Patel S, Cole AD, Nolan CM, et al. Pulmonary rehabilitation in bronchiectasis: a propensity-matched study. Eur Respir J 2019; 53: 1801264

14 British Thoracic Society. Quality Standards for Pulmonary Rehabilitation in Adults. British Thoracic Society Reports, Vol 6, No 2, 2014. London, British Thoracic Society, 2014. Available from: www.brit-thoracic.org.uk/ document-library/clinical-information/pulmonary-rehabilitation/bts-quality-standards-for-pulmonary-rehabilitationin-adults

15 McCarthy B, Casey D, Devane D, et al. Pulmonary rehabilitation for chronic obstructive pulmonary disease. Cochrane Database Syst Rev 2015: CD003793.

16 Spruit MA, Pitta F, Garvey C, et al. Differences in content and organisational aspects of pulmonary rehabilitation programmes. Eur Respir J 2014; 43: 1326-1337.

17 Bestall JC, Paul EA, Garrod R, et al. Usefulness of the Medical Research Council (MRC) dyspnoea scale as a measure of disability in patients with chronic obstructive pulmonary disease. Thorax 1999; 54: 581-586.

18 Chalmers JD, Goeminne P, Aliberti S, et al. The bronchiectasis severity index. An international derivation and validation study. Am J Respir Crit Care Med 2014; 189: 576-585.

19 Radovanovic D, Santus P, Blasi F, et al. A comprehensive approach to lung function in bronchiectasis. Respir Med 2018; 145: 120-129.

20 Aksamit TR, O'Donnell AE, Barker A, et al. Adult patients with bronchiectasis: a first look at the US bronchiectasis research registry. Chest 2017; 151: 982-992.

21 Vodanovich DA, Bicknell TJ, Holland AE, et al. Validity and reliability of the chronic respiratory disease questionnaire in elderly individuals with mild to moderate non-cystic fibrosis bronchiectasis. Respiration 2015; 90: 89-96. 
22 Spinou A, Fragkos KC, Lee KK, et al. The validity of health-related quality of life questionnaires in bronchiectasis: a systematic review and meta-analysis. Thorax 2016; 71: 683-694.

23 Nicolini A, Cardini F, Landucci N, et al. Effectiveness of treatment with high-frequency chest wall oscillation in patients with bronchiectasis. BMC Pulm Med 2013; 13: 21.

24 Murray MP, Pentland JL, Hill AT. A randomised crossover trial of chest physiotherapy in non-cystic fibrosis bronchiectasis. Eur Respir J 2009; 34: 1086-1092.

25 Vale F, Reardon JZ, ZuWallack RL. The long-term benefits of outpatient pulmonary rehabilitation on exercise endurance and quality of life. Chest 1993; 103: 42-45.

26 Troosters T, Gosselink R, Decramer M. Short- and long-term effects of outpatient rehabilitation in patients with chronic obstructive pulmonary disease: a randomized trial. Am J Med 2000; 109: 207-212.

27 Dudgeon EK, Crichton M, Chalmers JD. "The missing ingredient": the patient perspective of health related quality of life in bronchiectasis: a qualitative study. BMC Pulm Med 2018; 18: 81.

28 Hurst JR, Elborn JS, De Soyza A, et al. COPD-bronchiectasis overlap syndrome. The European respiratory journal. Eur Respir J 2015; 45: 310-313.

29 Jin J, Yu W, Li S, et al. Factors associated with bronchiectasis in patients with moderate-severe chronic obstructive pulmonary disease. Medicine 2016; 95: e4219. 